\title{
MODELOS HIPSOMÉTRICOS PARA UM POVOAMENTO EXPERIMENTAL DE TECA, PARÁ, BRASIL
}

\section{Deivison Venicio Souza ${ }^{1}$, Jamerson Rodrigo dos Prazeres Campos², Vanessa Maria Silva da $\mathrm{Cruz}^{3}$, Ana Paula Dalla Corte ${ }^{4}$, Carlos Roberto Sanquetta ${ }^{4}$ \\ ${ }^{1}$ Professor Mestre da Faculdade de Engenharia Florestal, Universidade Federal do \\ Pará, Altamira, PA, Brasil, e-mail: deivisonvs@ufpa.br \\ ${ }^{2}$ Professor substituto da Faculdade de Engenharia Florestal, Universidade Federal do Pará, Altamira, PA, Brasil. \\ ${ }^{3}$ Graduando em Engenharia Florestal, Universidade Federal do Pará, Tucuruí, PA, Brasil. \\ ${ }^{4}$ Professor Doutor do Departamento de Ciências Florestais, Universidade Federal do Paraná, Curitiba, PR, Brasil.}

Recebido em: 06/04/2018 - Aprovado em: 10/06/2018 - Publicado em: 20/06/2018 DOI: 10.18677/EnciBio_2018A59

\section{RESUMO}

Este estudo objetivou ajustar modelos de relação hipsométrica para um povoamento experimental de Tectona grandis L. f. implantado na Estação Experimental da Embrapa Amazônia Oriental, Km 23, BR-230, Altamira, Pará. Foram selecionadas 80 árvores-amostras, distribuídas em quatro classes diamétricas, as quais mediu-se o diâmetro a 1,30m (DAP) e a altura total $(\mathrm{H})$ de cada indivíduo, após a derruba. $\mathrm{O}$ conjunto de dados completos foi dividido em dados de ajuste $(80 \%)$ e validação $(20 \%)$. Testaram-se nove modelos hipsométricos tradicionais, tendo o DAP e suas transformações como variáveis independentes. As medidas de qualidade do ajuste usadas foram: Coeficiente de determinação ajustado ( $\left.R^{2} a j.\right)$, Erro padrão da estimativa em percentagem (Syx\%), Raiz do erro do quadrado médio (RMSE) e Critério de Informação de Akaike (AIC). Todas as pressuposições da regressão linear foram examinadas, assim como os indícios de multicolinearidade. A altura em sua forma natural obteve melhor correlação com o DAP quando este foi transformado para seu inverso e inverso ao quadrado. Uma simples transformação do diâmetro (1/DAP e 1/DAP2) foi capaz de aumentar a qualidade do ajuste de modelos com a altura na sua forma natural. Os modelos de Curtis e Assman foram indicados para a predição da altura de árvores de $T$. grandis, devido as suas melhores estatísticas de qualidade do ajuste. $\mathrm{O}$ emprego das equações ajustadas sobre novos dados confirmou a boa capacidade de generalização dos modelos.

PALAVRAS-CHAVE: Relação altura-diâmetro, regressão linear, Tectona grandis

\section{HYPSOMETRIC MODELS FOR AN EXPERIMENTAL SETTLEMENT OF TECA, PARÁ, BRAZIL}

\begin{abstract}
This study has the objective of adjust hypsometric relation models for an experimental settlement of Tectona grandis L. f. implanted on the Experimental Station of Embrapa Amazônia Oriental, Km 23, BR-230, Altamira, Pará. 80 sample trees was selected, distributed in four diametric classes, which were measured the
\end{abstract}


diameter of $1,30 \mathrm{~m}(\mathrm{DBH})$ and total height $(\mathrm{H})$ of each in subject, after take down. The complete data set was divided in adjustment data (80\%) and validation data $(20 \%)$. Nine standard hypsometric models were tested, having the $\mathrm{DBH}$ and its transformations as independent variables. The adjustment quality measures used were: Adjusted Coefficient of Determination ( $\left.R^{2} a d j.\right)$, Standard Error of the Estimate in percentage (Syx\%), Root Mean Square Deviation (RMSD) and Akaike Information Criterion (AIC). All linear regression assumptions were examined, as well as the indications of multicollinearity. Height an its natural form had a better correlation with $\mathrm{DBH}$ when this was transformed to its inverse and its squared inverse. One simple diameter transformation (1/DBH e $1 / \mathrm{DBH}^{2}$ ) was capable to increase the quality of models adjustment with the hight in its natural form. Curtis and Assman models were indicated for $\mathrm{T}$. grandis trees height prediction, due to their best quality statistics of adjustment. Te use of the adjusted equations over new data confirmed the good generalization capacity of the models.

KEYWORDS: hight-to-diameter ratio, linear regression, Tectona grandis

\section{INTRODUÇÃO}

A crescente demanda mundial por produtos madeireiros tem proporcionado a abertura de novos mercados e a busca de novas fontes produtoras, ampliando o horizonte para o setor florestal (ROSSI et al., 2011). Neste contexto, Tectona grandis L. f (Teca) tem se destacado no setor florestal, sobretudo, no mercado externo (SILVA et al., 2014), devido as suas qualidades silviculturais, industriais e comerciais (BEHLING et al., 2014), situando-se dentre as preferidas para o reflorestamento e para a produção de madeira sólida e de laminados (MOTTA et al., 2013).

A teca é uma espécie natural do continente asiático que é cultivada em várias regiões da África e das Américas (PELISSARI et al., 2014). O seu rápido crescimento em algumas regiões do Brasil, aliado a formação de um fuste retilíneo, tem estimulado o plantio em larga escala da espécie (LEITE et al., 2011), cujo uso tem se destinado à construção civil (portas, janelas, lambris, painéis e forros), produção de assoalhos e decks, móveis, embarcações e lâminas decorativas (ABRAF, 2013). No ano 2012, a superfície plantada com teca no Brasil foi de 67.329 hectares, possuindo produção sustentada de $1.009 .939 \mathrm{~m}^{3}$.ano-1, representando $0,4 \%$ do total da produção potencial e incremento médio anual de $15,0 \mathrm{~m}^{3}$. ha ${ }^{-1}$. Mato Grosso, Pará e Roraima destacaram-se com as maiores áreas plantadas (ABRAF, 2013).

Dada a importância da espécie no cenário nacional e internacional, em termos de produção madeireira, torna-se indispensável determinar com precisão a altura das árvores, isto porque, de acordo com Thiersch et al. (2013) a altura é uma importante variável para a estimativa do volume de madeira dos povoamentos e classificações destes quanto às suas produtividades. No entanto, a medição da altura de todas as árvores é uma atividade que demanda tempo, e que encarece o inventário florestal, além disso, está sujeita a erros devido às dificuldades de campo (SANQUETTA et al., 2014).

Desta forma, para contornar esses problemas tem-se como prática o desenvolvimento de equações hipsométricas, através das quais seja possível estimar as alturas das demais árvores (THIERSCH et al., 2013). Assim, para o ajuste de modelos hipsométricos tradicionais para florestas plantadas, mede-se o diâmetro a 1,30 m do solo (DAP) de todas as árvores na parcela amostral, porém selecionam-se apenas algumas árvores para a mensuração da altura (RIBEIRO et 
al., 2010). Em seguida, de posse dos pares de valores altura-diâmetro, seleciona-se a equação hipsométrica mais acurada para estimativa da variável dependente, tendo por base diversos parâmetros estatísticos. De posse do modelo selecionado, podem-se estimar as alturas das árvores que tiveram apenas o DAP medido em campo.

Ribeiro et al. (2010) afirmam que diversos fatores devem ser considerados para a construção adequada de modelos hipsométricos, tais como posição sociológica, região, idade, densidade do plantio e práticas silviculturais. Em nível de espécie, Tonini et al. (2005) destacaram o comportamento distinto da curva hipsométrica, indicando a necessidade de se realizar modelagem por espécie.

Este estudo teve como objetivo ajustar e selecionar modelos hipsométricos tradicionais com maior acuracidade para estimar a variável altura para um povoamento experimental de $T$. grandis localizado em Altamira, Sudoeste do Pará.

\section{MATERIAL E MÉTODOS}

\section{Área de estudo e coleta de dados}

O estudo foi realizado em um povoamento plantado de Tectona grandis L. f. localizado na estação experimental da Embrapa Amazônia Oriental, situada às margens da BR-230 (Rodovia Transamazônica), a $23 \mathrm{~km}$ do município de Altamira, Sudoeste do estado do Pará. O povoamento foi conduzido sem tratamentos silviculturais, e possui um total de 546 indivíduos, com idades variando entre 5 e 14 anos, distribuídos em uma área de $3.276 \mathrm{~m}^{2}$, e conduzidos sob o espaçamento $3 \mathrm{~m} \mathrm{x}$ $2 \mathrm{~m}$. O povoamento está sob a coordenada geográfica: $03^{\circ} 16^{\prime} 10,96$ " latitude e $52^{\circ} 23^{\prime} 42,58^{\prime \prime}$ longitude.

Em virtude de sua extensão, o município apresenta clima do tipo equatorial Am e Aw, conforme classificação de Köppen. O clima Am, é predominante da parte norte, onde apresenta temperaturas médias de $26^{\circ} \mathrm{C}$, e precipitação anual em torno de $1.680 \mathrm{~mm}$, sendo que os meses mais chuvosos vão de dezembro a maio e, os menos chuvosos, de junho a novembro. Ao sul, predomina o clima Aw, que passa por uma transição até alcançar o tipo savana. O excedente de água ocorre entre fevereiro e abril e a maior deficiência, em setembro. Os solos predominantes são: i) Latossolo Amarelo texturas média e argilosa; ii) Latossolo Vermelho-Amarelo texturas média e argilosa; iii) Podzólico Vermelho-Amarelo texturas média e argilosa e equivalente eutrófico terra roxa estruturada (IDESP, 2014).

Foram selecionadas 80 árvores-amostras, distribuídas em quatro classes diamétricas. Inicialmente, de cada árvore mediu-se o DAP e, em seguida, derrubaram-se os indivíduos para a medição da altura total $(\mathrm{H})$. As medições foram realizadas com o emprego de uma suta mecânica e fita métrica. Neste estudo, o conjunto de dados completos foi dividido em dados de ajuste (80\%) e validação $(20 \%)$.

\section{Modelos hipsométricos e critérios de seleção}

Foram ajustados nove modelos hipsométricos tradicionais, bastante disseminados na literatura florestal (quadro 1). A escolha do melhor modelo de regressão baseou-se nos critérios estatísticos: Coeficiente de determinação ajustado $\left(R^{2}\right.$ aj.), Erro padrão da estimativa em percentagem (Syx\%), Raiz do erro do quadrado médio (RMSE) e Critério de Informação de Akaike (AIC). O teste $t$-Student $(\alpha=0,05)$ foi utilizado para avaliar a significância dos coeficientes da regressão. 
QUADRO 1. Modelos ajustados para um plantio experimental de T. grandis, Pará, Brasil.

\begin{tabular}{lll}
\hline Eq. & Modelo & Autor \\
\hline $\mathbf{1}$ & $h=\beta_{0}+\beta_{1} d+\varepsilon_{i}$ & Linha Reta $^{1}$ \\
$\mathbf{2}$ & $h=\beta_{0}+\beta_{1} 1 / d+\varepsilon_{i}$ & Curtis $(1967)^{2}$ \\
$\mathbf{3}$ & $h=\beta_{0}+\beta_{1} d+\beta_{2} d^{2}+\varepsilon_{i}$ & Trorey $(1932)^{2}$ \\
$\mathbf{4}$ & $h=\beta_{0}+\beta_{1} 1 / d+\beta_{2} d+\varepsilon_{i}$ & Sanquetta et al. (2013) \\
$\mathbf{5}$ & $h=\beta_{0}+\beta_{1} 1 / d^{2}+\varepsilon_{i}$ & Assmann \\
$\mathbf{6}$ & $h=\beta_{0}+\beta_{1} \operatorname{Ln}(d)+\varepsilon_{i}$ & Henricksen $(1950)^{2}$ \\
$\mathbf{7}$ & $\operatorname{Ln}(h)=\beta_{0}+\beta_{1} \log (d)+\varepsilon_{i}$ & Stoffels \& Soest $(1953)^{2}$ \\
$\mathbf{8}$ & $d^{2} / h=\beta_{0}+\beta_{1} d+\beta_{2} d^{2}+\varepsilon_{i}$ & Prodan $(1965)^{1}$ \\
$\mathbf{9}$ & $\operatorname{Ln}(h)=\beta_{0}+\beta_{1} \operatorname{Ln}(d)+\beta_{2}(1 / d)+\varepsilon_{i}$ & Silva $(1980)$ \\
\hline
\end{tabular}

Em que: $\mathrm{h}=$ altura total da árvore $(\mathrm{m}) ; \mathrm{d}=$ diâmetro medido a 1,30 do solo $(\mathrm{cm}) ; \mathrm{Ln}=$ logaritmo neperiano; $\beta_{0}, \beta_{1}, \beta_{2}=$ coeficientes da regressão; e $\varepsilon_{\mathrm{i}}=$ termo de erro aleatório. Fonte: ${ }^{1}$ Machado et al (2008); ${ }^{2}$ Figueiredo Filho et al. (2010); ${ }^{3}$ Sena et al. (2015).

Os resíduos da regressão linear foram julgados quanto às pressuposições de homocedasticidade (Breusch-Pagan, $\alpha=0,05$ ), autocorrelação (Durbin-Watson, $\alpha=0,05$ ) e normalidade (Shapiro-Wilk, $\alpha=0,05$ ). Adicionalmente, para confirmar o atendimento as pressuposições de homocedasticidade e normalidade plotou-se os gráficos de resíduos versus valores ajustados (escala da variável dependente) e, também o gráfico de Papel de Probabilidade Normal (Q-Qplot). O grau de confundimento entre variáveis preditora foi verificado por meio da estatística Variance Inflation Factor - (VIF), para detectar indícios de problemas de multicolinearidade.

Todas as análises foram feitas usando diversos pacotes do ambiente estatístico $R$, versão 3.3.3. A matriz de correlações de Pearson e o ajuste dos modelos de regressão linear foram realizados com auxílio do pacote "stats" disponível no R-base. Além disso, foram utilizadas funções de diversos pacotes para análises estatísticas básicas (WUERTZ et al., 2014; HARRELL Jr., 2016), análise de pressuposições da regressão linear (ZEILEIS; HOTHORN, 2002; FOX; WEISBERG, 2011), indícios de multicolinearidade (FARAWAY, 2016) e métricas de desempenho (HAMNER; FRASCO, 2017).

\section{RESULTADOS E DISCUSSÃO}

$\mathrm{Na}$ Tabela 1 está a estatística descritiva para os conjuntos de dados das variáveis dendrométricas usadas no ajuste e validação da relação hipsométrica. As árvores utilizadas no ajuste dos modelos tiveram DAP variando de $6,60 \mathrm{~cm}$ a $32,60 \mathrm{~cm}$, com uma amplitude interquartil de $5,80 \mathrm{~cm}$, denotando que $50 \%$ dos diâmetros estiveram entre $12,30 \mathrm{~cm}\left(Q_{1}\right)$ e $18,10 \mathrm{~cm}\left(Q_{3}\right)$.

TABELA 1. Estatística descritiva para as variáveis dendrométricas para um plantio de $T$. grandis, Pará, Brasil.

\begin{tabular}{|c|c|c|c|c|c|c|c|c|c|c|c|}
\hline \multicolumn{12}{|l|}{ Ajuste } \\
\hline $\begin{array}{l}\text { DAP (cm) } \\
\mathrm{H}(\mathrm{m})\end{array}$ & $\begin{array}{l}n \\
65\end{array}$ & $\begin{array}{l}\text { Me } \\
15,22 \\
16,64\end{array}$ & $\begin{array}{l}\mathbf{Q}_{0.25} \\
12,30 \\
14,88\end{array}$ & $\begin{array}{l}\text { Md } \\
14,60 \\
17,20\end{array}$ & $\begin{array}{l}\mathbf{Q}_{0.75} \\
18,10 \\
19,00\end{array}$ & $\begin{array}{l}\text { Sd } \\
4,99 \\
3,34\end{array}$ & $\begin{array}{l}\mathbf{g}_{1} \\
0,54 \\
-0,87\end{array}$ & $\begin{array}{l}\mathbf{g}_{\mathbf{2}} \\
1,13 \\
0,83\end{array}$ & $\begin{array}{l}\text { Min. } \\
6,60 \\
6,87\end{array}$ & $\begin{array}{l}\text { Max. } \\
32,60 \\
22,80\end{array}$ & $\begin{array}{l}\text { IQR } \\
5,80 \\
4,12\end{array}$ \\
\hline \multicolumn{12}{|l|}{ Validação } \\
\hline $\begin{array}{l}\text { DAP (cm) } \\
\mathrm{H}(\mathrm{m})\end{array}$ & 15 & $\begin{array}{l}15,09 \\
16,39\end{array}$ & $\begin{array}{l}12,85 \\
15,05\end{array}$ & $\begin{array}{l}14,70 \\
18,15\end{array}$ & $\begin{array}{l}18,10 \\
18,58\end{array}$ & $\begin{array}{l}4,91 \\
3,81\end{array}$ & $\begin{array}{l}-0,09 \\
-1,19\end{array}$ & $\begin{array}{l}-0,43 \\
0,08\end{array}$ & $\begin{array}{l}7,00 \\
8,60\end{array}$ & $\begin{array}{l}23,70 \\
20,10\end{array}$ & $\begin{array}{l}5,25 \\
3,53\end{array}$ \\
\hline
\end{tabular}

Em que: $\mathrm{N}$ = quantidade de indivíduos; $\mathrm{Me}=$ média aritmética; $\mathrm{Q}_{0.25}=$ primeiro quartil; Md = mediana; $\mathrm{Q}_{0.75}=$ terceiro quartil; $\mathrm{Sd}=$ desvio padrão; $\mathrm{g}_{1}=$ coeficiente de assimetria de Fisher; $\mathrm{g}_{2}=$ coeficiente de achatamento de Fisher; Min. = mínimo; Max. = máximo; IQR = amplitude interquartil. 
A variabilidade dos DAP em relação à média foi alta $(C V=32,79 \%)$ indicando conjuntos de dados heterogêneos, sobretudo, por se tratar de um povoamento experimental. A altura das árvores variou de $6,87 \mathrm{~m}$ a $22,80 \mathrm{~m}$. A variação em torno da média foi de aproximadamente $20 \%$. A divisão dos dados assegurou a representatividade das árvores dentro de todas as classes diamétricas, além de garantir que as propriedades estatísticas (média e desvio padrão) fossem semelhantes entre si.

Foi realizada a análise da correlação de Pearson entre as variáveis dependentes e independentes e entre as variáveis independentes. Foram identificadas correlações positivas ou negativas, significativas ao nível de $1 \%$ de probabilidade através do teste $t$-Student (Tabela 2).

A altura em sua forma natural obteve melhor correlação com o DAP quando este foi transformado para seu inverso e inverso ao quadrado, respectivamente. A variável dependente quando transformada para a forma $\mathrm{DAP}^{2} / \mathrm{H}$ mostrou-se fortemente correlacionada com a variável DAP e DAP ${ }^{2}$ (Prodan). Porém, DAP e DAP $^{2}$ estiveram fortemente correlacionadas $(r=0,97)$.

TABELA 2. Matriz de correlação de Pearson entre variáveis dependentes e independentes e entre as variáveis independentes das equações hipsométricas ajustadas para um plantio de T. grandis, Pará, Brasil.

\begin{tabular}{|c|c|c|c|c|c|c|}
\hline \multirow{2}{*}{ Variáveis } & & \multicolumn{5}{|c|}{ Independentes } \\
\hline & & $\overline{D A P}$ & $\mathrm{DAP}^{2}$ & $\operatorname{Ln}(\mathrm{DAP})$ & 1/DAP & $1 / \mathrm{DAP}^{2}$ \\
\hline \multirow{3}{*}{ Dependentes } & $\mathrm{H}$ & $0,76^{* *}$ & $0,66^{* *}$ & $0,83^{* *}$ & $-0,86^{* *}$ & $-0,85^{* *}$ \\
\hline & $\operatorname{Ln}(\mathrm{H})$ & & & $0,82^{* *}$ & $-0,87^{* *}$ & \\
\hline & $\mathrm{DAP}^{2} / \mathrm{H}$ & $0,95^{* *}$ & $0,97^{* \star}$ & & & \\
\hline Independente & DAP & & $0,97^{* *}$ & & $-0,90^{* *}$ & \\
\hline $\mathbf{s}$ & 1/DAP & & & $-0,98^{* *}$ & & \\
\hline
\end{tabular}

${ }^{* *}$ significativo através do teste $t$-Student ao nível de $1 \%$ de probabilidade $(p \leq 0,001)$.

Sanquetta et al. (2014), estudando a relação hipsométrica em povoamentos de Acacia mearnsii, reportaram tendências semelhantes a este estudo, ao observarem que a relação da altura em sua forma natural com o diâmetro foi significativa ao nível de $5 \%$ de probabilidade $(p \leq 0,05)$, e ainda, que a transformação da variável DAP para seu inverso e inverso ao quadrado ocasionou uma melhora na relação com a altura.

Indícios de existência de multicolinearidade foram detectados para os modelos de Trorey, Prodan, Sanquetta et al. (2013) e Silva (1980), cujas correlações entre as variáveis preditoras foram superiores a $90 \%$. A estatística Variance Inflation Factor - (VIF) foi usada para detectar a existência de confundimento entre preditoras (multicolinearidade). Assim, a estatística VIF confirmou haver efeitos graves de multicolinearidade para os preditores de Trorey, Prodan e Silva (1980) (VIF >10) (Tabela 3).

A estatística F-Snedecor foi significativa ao nível de $1 \%$ de probabilidade $(\alpha=$ $0,01)$. O teste Durbin-Watson $(\alpha=0,05)$ não apontou indícios de autocorrelação dos resíduos para os modelos. Os modelos de Trorey, Sanquetta et al. (2013) e Prodan mostraram pelo menos um coeficiente não diferente de zero, através da estatística $t$ Student $(\alpha=0,05)$.

As equações conseguiram explicar mais de $60 \%$ das variações ocorridas na variável predita, com exceção do modelo de Linha Reta. Assim, ratifica-se que usar exclusivamente do diâmetro, em sua escala natural, para prever a altura não é uma 
boa opção. Os scores de RMSE variaram pouco entre modelos ficando em torno de $2 m$.

Sanquetta et al. (2015) ajustando modelos de relação hipsométrica em plantios jovens de Tectona grandis, Redenção, Pará, observaram valores superiores para $\mathrm{R}^{2}$ aj. e inferiores para Syx (\%) em relação a este estudo, para os modelos de Henriksen $\left(R^{2} a j .=88,0 \%\right.$ e Syx $=11,83$ e Stoffels $\left(R^{2} a j .=88,0 \%\right.$ e Syx $\left.=11,83 \%\right)$.

Pereira et al. (2014) ajustando modelos hipsométricos para um plantio de Tectona grandis, aos 13 anos de idade, Moju, Pará, encontraram resultados inferiores de $\mathrm{R}^{2}$ aj. e Syx (\%) para os modelos em comum a este estudo. Os modelos de Stoffels, Henricksen, Trorey tiveram $\left(R^{2} a j .=46,9 \%\right.$ e Syx $\left.=16,7 \%\right),\left(R^{2} a j .=\right.$ $44,9 \%$, Syx $=16,8 \%),\left(R^{2}\right.$ aj. $=44,8 \%$, Syx=16,8\%), respectivamente.

$\mathrm{O}$ diagnóstico da homocedasticidade e normalidade dos resíduos (Figura 1) foi feito apenas para os modelos Curtis, Assmann, Henriksen e Stoffels, haja vista não apresentarem coeficientes da regressão nulos $(\alpha=0,05)$ e, ainda não terem sidos desqualificados, a priori, por problemas de coeficientes da regressão não significativos $(\alpha=0,05)$ ou de graves indícios de multicolinearidade (no caso de regressões múltiplas).

TABELA 3. Coeficientes e estatísticas de qualidade dos ajustes de modelos hipsométricos para um plantio experimental de T. grandis, Pará, Brasil.

\begin{tabular}{|c|c|c|c|c|c|c|c|c|c|}
\hline \multirow{2}{*}{ Eq. } & \multicolumn{3}{|c|}{ Coeficientes } & \multirow{2}{*}{$\mathbf{R}_{\text {aj. }}$} & \multirow{2}{*}{$\mathrm{S}_{\mathrm{yx}} \%$} & \multirow{2}{*}{ RMSE } & \multirow{2}{*}{ AIC } & \multirow{2}{*}{ VIF } & \multirow{2}{*}{ FM } \\
\hline & $\beta_{0}$ & $\boldsymbol{\beta}_{1}$ & $\beta_{2}$ & & & & & & \\
\hline 1 & 8,87899 & 0,50957 & & 0,5725 & 13,03 & 2,1514 & 290,0582 & & \\
\hline 2 & 24,0693 & $-100,4334$ & & 0,7319 & 10,32 & 1,7038 & 259,7339 & & \\
\hline 3 & $1,8236^{\mathrm{ns}}$ & 1,4538 & $-0,0286$ & 0,6889 & 11,12 & 1,8206 & 270,3546 & 17,65 & \\
\hline 4 & 25,3721 & $-108,0755$ & $-0,0484^{\text {ns }}$ & 0,7286 & 10,38 & 1,7007 & 261,4949 & 5,39 & \\
\hline 5 & 19,9697 & $-530,7698$ & & 0,7197 & 10,55 & 1,7422 & 262,6322 & & \\
\hline 6 & $-4,6514$ & 7,9831 & & 0,6862 & 11,17 & 1,8433 & 269,9637 & & \\
\hline 7 & 1,2957 & 0,5593 & & 0,6728 & 12,43 & 2,0484 & $-71,64349$ & & 1,009 \\
\hline 8 & $2,0842^{\text {ns }}$ & $0,0837^{\mathrm{ns}}$ & 0,0437 & 0,9462 & 39,35 & 1,7407 & 270,5401 & 17,65 & \\
\hline 9 & 4,8338 & $-0,4263$ & $-12,2928$ & 0,7689 & 10,44 & 1,7111 & $-93,29853$ & 22,42 & 1,006 \\
\hline
\end{tabular}

Eq. = Equações; $\beta_{0}, \beta_{1}$ e $\beta_{2}=$ coeficientes da regressão; $R^{2}$ aj. = Coeficiente de determinação ajustado; Syx\%= Erro padrão da estimativa recalculado, em \%; RMSE = Raiz quadrada do erro médio; AIC = Critério de Informação de Akaike; VIF = fator de inflação de variância determinado para cada coeficiente da regressão (na sequência do modelo ajustado $\left(\beta_{1}, \beta_{2}, . ., \beta_{n}\right) ; F M=$ Fator de Meyer; ns $=$ não significativo através do teste $t$-Student a $5 \%$ de probabilidade $(\alpha=0,05)$. 


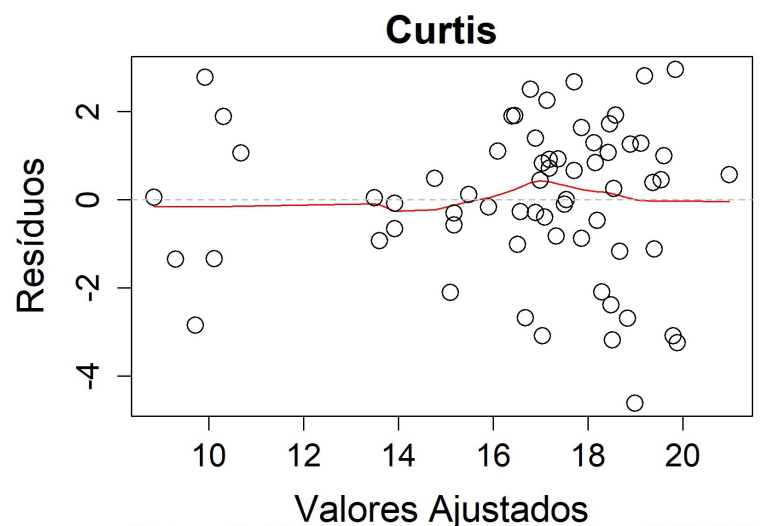

$($ Breusch-Pagan $=1,2898 ;$ p-valor $=0,2561)$

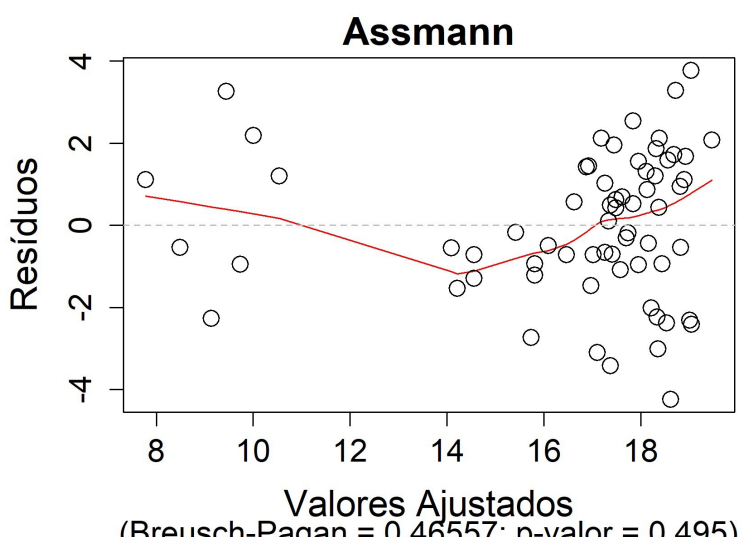

(Breusch-Pagan = 0,46557; p-valor $=0,495)$
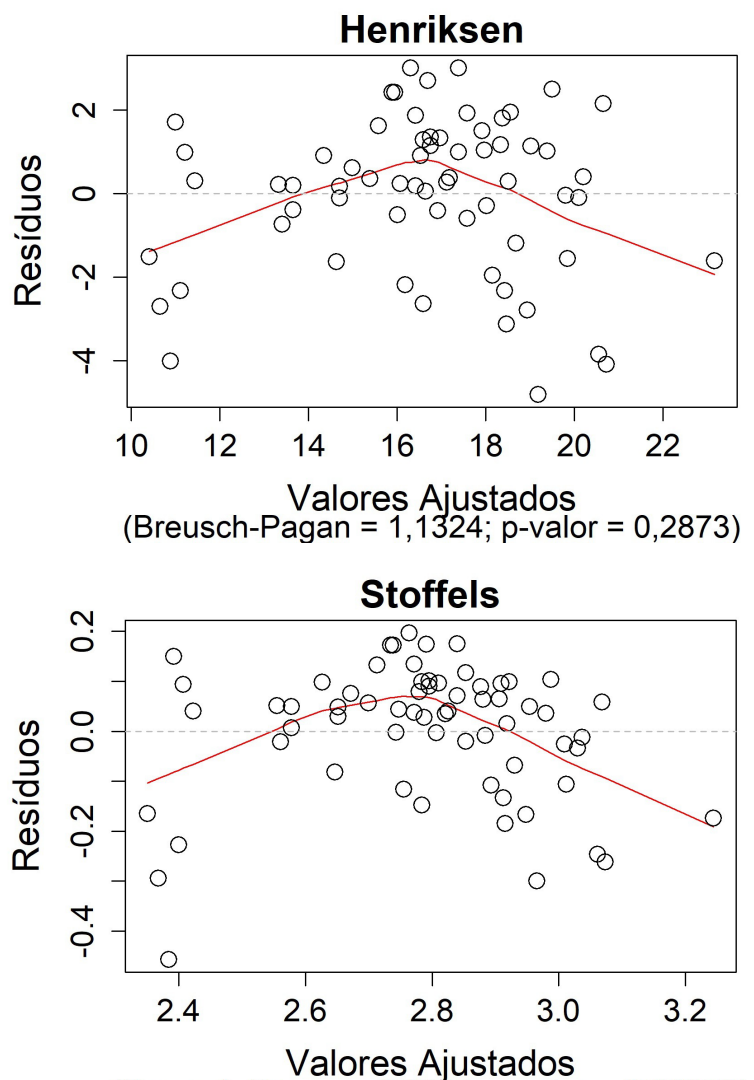

$($ Breusch-Pagan $=2,9911 ; p$-valor $=0,08372)$

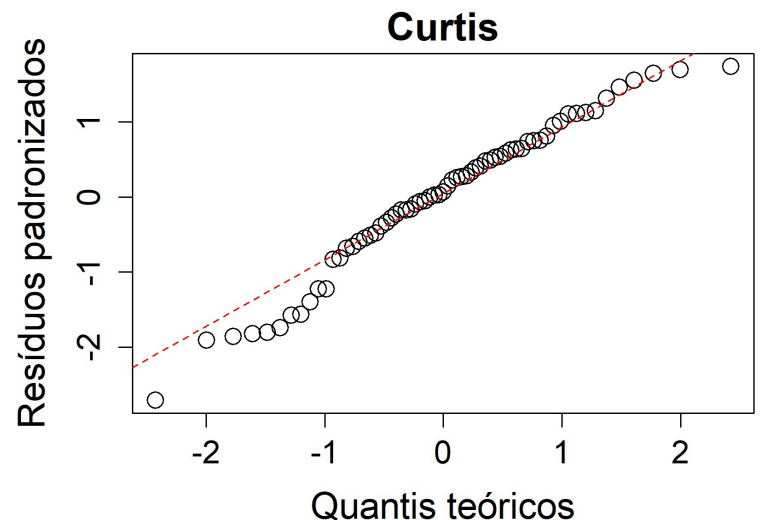

(Shapiro-Wilk =0,96831; p-valor $=0,09395$ )

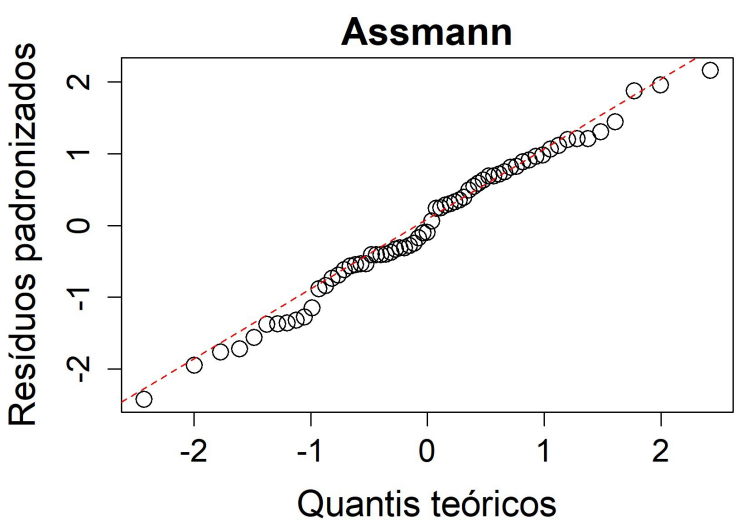

(Shapiro-Wilk $=0,98674 ;$-valor $=0,7157$ )

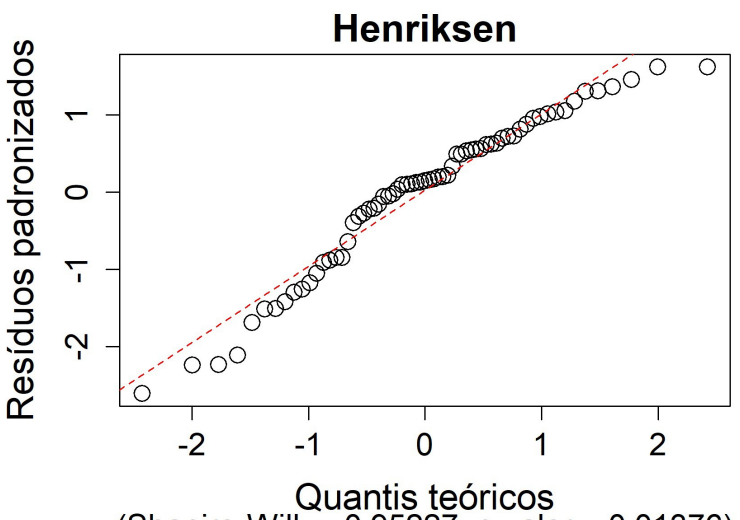

(Shapiro-Wilk = 0,95227; $\mathrm{p}$-valor $=0,01376$ )

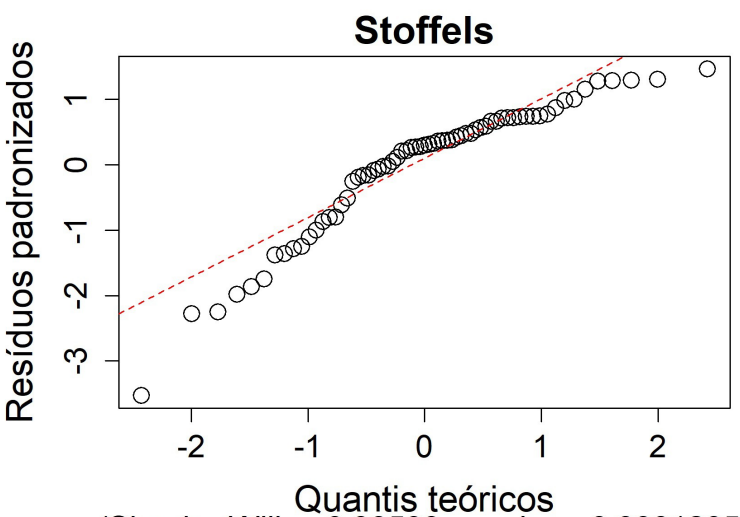

(Shapiro-Wilk = 0,90599; $p$-valor $=0,0001205$

FIGURA 1: Resíduos versus valores ajustados (escala da variável dependente) e Papel de Probabilidade Normal (Q-Qplot) para as equações sem autocorrelação de resíduos, multicolinearidade e coeficientes da regressão nulos. 
As equações de Henriksen e Stoffels não atenderam à pressuposição de normalidade dos resíduos, portanto, foram insatisfatórios. Os testes de BreuschPagan e Shapiro-Wilk atestaram homocedasticidade de variâncias e normalidade de resíduos $(\alpha=0,05)$ para Curtis e Assmann.

A equação ajustada de Curtis revela um padrão de variância mais homogêneo, em torno da média zero, conforme indicado pela curva de suavização (linha vermelha). Além disso, as estatísticas de qualidade do ajuste foram ligeiramente melhores para Curtis quando comparadas àquelas obtidas para Assmann. Por fim, constatou-se que uma simples transformação do diâmetro (1/DAP e 1/DAP ${ }^{2}$ ) foi capaz de aumentar a qualidade do ajuste de modelos com a altura na sua forma natural.

Ambos os modelos (Curtis e Assman) tiveram satisfatório desempenho sobre novos dados, com elevada correlação entre os valores previstos e observados (Figura 2). Os scores de RMSE foram baixos e corroboraram com a estimativa de desempenho obtida no procedimento de ajuste por meio do método de mínimos quadrados ordinários (ver tabela 2).
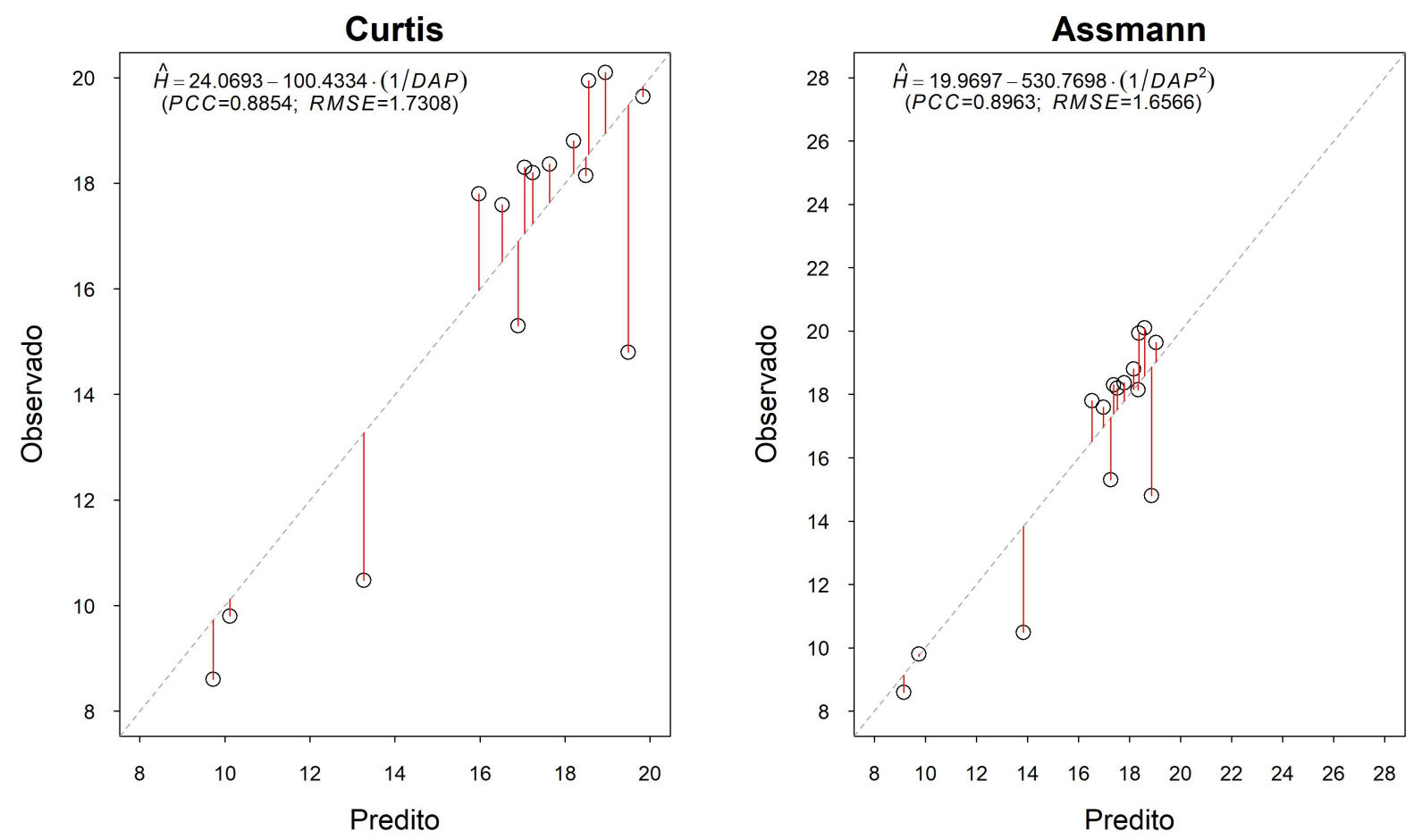

FIGURA 2. Valores observados versus preditos para o conjunto de dados de validação usando os modelos de Curtis e Assman. Em que: PCC = Coeficiente de correlação de Pearson; RMSE = Raiz quadrada do erro médio; DAP = diâmetro a $1,30 \mathrm{~m}$ do solo $(\mathrm{cm}) ; \widehat{H}=$ altura estimada $(\mathrm{m})$.

\section{CONCLUSÃO}

Os modelos lineares (Curtis e Assman) mostraram as melhores medidas de desempenho no ajuste, com atendimento a todas as pressuposições da regressão linear. A qualidade preditiva dos modelos foi confirmada no conjunto de validação, com dados independentes do ajuste. 


\section{AGRADECIMENTOS}

Os autores expressam seus agradecimentos à Empresa Brasileira de Pesquisa Agropecuária (Embrapa) pela cessão de sua área experimental de $T$. grandis para a realização desta pesquisa científica.

\section{REFERÊNCIAS}

ABRAF. Anuário estatístico da ABRAF 2013 - ano base 2012. Brasília: ABRAF, 2013.

BEHLING, M.; NEVES, J. C. L.; BARROS, N. F.; KISHIMOTO, C. B.; SMIT, L. Eficiência de utilização de nutrientes para formação de raízes finas e médias em povoamento de teca. Revista Árvore, Viçosa-MG, v.38, n.5, p.837-846, 2014. Disponível em: <http://dx.doi.org/10.1590/S0100-67622014000500008>. doi: 10.1590/S0100-67622014000500008

CURTIS, R. O. Height-Diameter and Height-Diameter-Age Equations for SecondGrowth Douglas-Fir. Forestry Science, v.13, n.4, p.365-375, 1967. Disponível em: $<$ https://doi.org/10.1093/forestscience/13.4.365>.

doi: 10.1093/forestscience/13.4.365

FARAWAY, J. Faraway: Functions and Datasets for Books by Julian Faraway. R package version 1.0.7. 2016. Disponível em: <https://cran.rproject.org/web/packages/faraway/faraway.pdf>.

FIGUEIREDO FILHO, A., DIAS, A. N., KOHLER, S. V., VERUSSA, A. A., CHIQUETTO, A. L. Evolução da relação hipsométrica em plantios de Araucaria angustifolia no centro-sul do estado do Paraná. Cerne, Lavras, v.16, n.3, p.347-357, 2010. Disponível em: <https://dx.doi.org/10.1590/S0104-77602010000300010>. doi: 10.1590/S0104-77602010000300010

FOX, J.; WEISBERG, S. An $\{R\}$ Companion to Applied Regression. v.1 2.ed. Thousand Oaks CA: Sage. ISBN-10: 141297514X. 2011. 472p

HAMNER, B.; FRASCO, M. Metrics: Evaluation Metrics for Machine Learning. R package version 0.1.3. 2017. Disponível em: <https://CRAN.Rproject.org/package=Metrics $>$.

HARRELL Jr, F. E. With contributions from Charles Dupont and many others. Hmisc: Harrell Miscellaneous. R package version 4.0-2. 2016. Disponível em: $<$ https://CRAN.R-project.org/package=Hmisc $>$.

IDESP - Instituto de Desenvolvimento Econômico, Social e Ambiental do Pará. Estatística Municipal: Relatório Técnico. Altamira, 2014.

LEITE, H. G.; OLIVEIRA-NETO, R. R.; MONTE, M. A.; FARDIN, L.; ALCANTARA, A. M.; BINOTI, M. L. M. S.; CASTRO, R. V. O. Modelo de afilamento de cerne de Tectona grandis L. f. Scientia Forestalis, Piracicaba, v.39, n.89, p.053-059, mar. 2011. Disponível em: < http://www.ipef.br/publicacoes/scientia/nr89/cap05.pdf>. 
MACHADO, S. A., NASCIMENTO, R. G. M., AUGUSTYNCZIK, A. L. D., SILVA, L. C. R., FIGURA, M. A., PEREIRA, E. M., TÉO, S. J. Comportamento da relação hipsométrica de Araucaria angustifolia no capão da Engenharia Florestal da UFPR. Pesquisa Florestal Brasileira, v.5, n.56, p.5-16, 2008. Disponível em: < https://ainfo.cnptia.embrapa.br/digital/bitstream/CNPF-200909/44379/1/1_S_A_Machado2.pdf>.

MOTTA, J. P.; OLIVEIRA, J. T. S.; PAES, J. B.; ALVES, R. C.; DAMBROZ, G. B. V. Resistência natural da madeira de Tectona grandis em ensaio de laboratório. Ciência Rural, Santa Maria, v.43, n.8, p.1393-1398, ago/2013. Disponível em: < http://dx.doi.org/10.1590/S0103-84782013005000097>. doi: 10.1590/S010384782013005000097

PELISSARI, A. L.; GUIMARÃES, P. P.; BEHLING, A.; EBLING, Â. A. Cultivo da teca: características da espécie para implantação e condução de povoamentos florestais. Agrarian Academy, Centro Científico Conhecer, Goiânia, v.1, n.01; p. 128. 2014. Disponivel em: <http://www.conhecer.org.br/Agrarian \%20Academy/2014a/cultivo.pdf>. doi: 10.18677/Agrarian Academy 2014_011

PEREIRA, K. D.; PAIXÃO, M. V. C.; MONTEIRO, C. W. B.; LAURIDO, F. T.; FERREIRA, P. S. T. Ajuste de modelos hipsométricos para árvores de Tectona grandis L. f. no município de Mojú, Pará. Enciclopédia biosfera, Centro Científico Conhecer, Goiânia, v.10, n.18; 2014. Disponível em: < http://www.conhecer.org.br/enciclop/2014a/agrar.htm>.

RIBEIRO, A.; FERRAZ FILHO, A. C.; MELLO, J. M.; FERREIRA, M. Z.; LISBOA, P. M. M.; SCOLFORO, J. R. S. Estratégias e metodologias de ajuste de modelos hipsométricos em plantios de Eucalyptus sp. Cerne, Lavras, v. 16, n. 1, p.22-31, jan./mar. 2010. Disponível em: <http://dx.doi.org/10.1590/S010477602010000100003.>. doi: 10.1590/S0104-77602010000100003

ROSSI, A. S.; DRESCHER, R.; PELISSARI, A. L.; LANSSANOVA, L. R. Relação hipsométrica e crescimento de Tectona grandis L. f. no município de Monte Dourado, Pará. Scientia Forestalis, Piracicaba, v. 39, n. 91, p. 301-307, set. 2011.

SANQUeTTA, C. R.; CORTE, A. P. D.; ROGLIN. A.; PIMENTEL. A. Relações diâmetro-altura para espécies lenhosas em um fragmento de Floresta Ombrófila Mista no Sul do Paraná. Iheringia Série Botânica, Porto Alegre, v. 68, n. 1, p. 103114, junho 2013. Disponível em: < http://www.fzb.rs.gov.br/upload/20140328094646ih68_1_p103_114.pdf >.

SANQUETTA, C. R.; BEHLING, A.; CORTE, A. P. D.; RUZA, M. S.; SIMON, A.; JOSÉ, J. F. B. S. Relação hipsométrica em inventários pré-corte em povoamentos de Acacia mearnsii De Wild. Científica, Jaboticabal, v.42, n.1, p.80-90, 2014. Disponível em: < http://cientifica.org.br/index.php/cientifica/article/viewFile/19845529.2014v42n1p080-090/286>.

SANQUETTA, M. N. I.; SANQUETTA, C. R.; MOGNON, F.; CORTE, A. P. D.; RODRIGUES, A. L.; MAAS, G. C. B. Ajuste de equações hipsométricas para a estimação da altura total de indivíduos jovens de teca. Científica, Jaboticabal, v.43, 
n.4, p.400-406, 2015. Disponível em: < http://cientifica.org.br/index.php/cientifica/article/view/741>.

SENA, A. L. M.; SILVA NETO, A. J.; OLIVEIRA, G. M. V.; CALEGARIO, N. Modelos lineares e não lineares com uso de covariantes para relação hipsométrica de duas espécies de Pinus tropicais. Ciência Florestal, 25(4), 969-980, 2015. Disponível em: <https://dx.doi.org/10.5902/1980509820651>. doi: 10.5902/1980509820651

SILVA, F. R.; SILVA, V. S. M.; MIRANDA, S. O. Crescimento de Tectona grandis em um uma plantação no município de Alta Floresta, Mato Grosso. Floresta, Curitiba, PR, v. 44, n. 4, p. 577-588, out./dez. 2014.

TONINI, H.; ARCO-VERDE, M. F.; SÁ, S. P. P de. Dendrometria de espécies nativas em plantios homogêneos no Estado de Roraima - Andiroba (Carapa guianensis Aubl), Castanha-do-Brasil (Bertholletia excelsa Bonpl.), Ipê-roxo (Tabebuia avellanedae Lorentz ex Griseb) e Jatobá (Hymenaea courbaril L.). Acta Amazônica, v. 35, n.3, p.353 - 362, 2005. Disponível em: <http://dx.doi.org/10.1590/S004459672005000300008>. doi: 10.1590/S0044-59672005000300008

THIERSCH, C. R.; ANDRADE, M. G.; MOREIRA, M. F. B.; LOIBEL, S. Estimativa da relação hipsométrica em clones de Eucalyptus sp. com o modelo de curtis ajustado por métodos bayesianos empíricos. Revista Árvore, Viçosa-MG, v.37, n.1, p.01-08, 2013. Disponível em: <http://dx.doi.org/10.1590/S0100-67622013000100001.>. doi: 10.1590/S0100-67622013000100001

WUERTZ, D.; SETZ, T.; CHALABI, Y. Rmetrics Core Team, fBasics: Rmetrics Markets and Basic Statistics. R package version 3011.87. 2014.

ZEILEIS, A.; HOTHORN, T. Diagnostic Checking in Regression Relationships. $R$ News 2(3), 7-10. 2002. Disponível em: <http://CRAN.R-project.org/doc/Rnews/>. 